\title{
Does Gestalt hearing exist?
}

\author{
Timothy Chenette ${ }^{1 \dagger}$ \\ Alexandra Phillips ${ }^{2}$ \\ Emily Wood ${ }^{3}$ \\ ${ }^{1,2,3}$ Utah State University, Logan, Utah, United States \\ $\dagger$ Corresponding author: timothy.chenette@usu.edu \\ Published 16 December 2021; https://doi.org/10.18061/FDMC.2021.0028 \\ Author video presentation and/or other conference material: https://doi.org/10.17605/OSF.IO/X62CZ
}

\begin{abstract}
Karpinski 2000 describes "Gestalt hearing," identifying chords instantly and holistically, as the ideal endpoint of training in harmonic dictation. Yet the subdominant chord (e.g.) is not a single object but a collection of objects that differ in timbre, texture, inversion, spacing, etc. Is it really possible to become so acquainted with this group that one perceives it as a Gestalt? If so, what experiences and abilities are necessary to develop Gestalt hearing? This article draws on relevant results from an observational, exploratory study of harmonic listening $(N=73)$ to suggest preliminary answers to these questions and paths for future research in this area. Our results suggest that Gestalt hearing, if it exists, may not be available in college-level instruction.
\end{abstract}

KEYWORDS: harmonic dictation, pedagogy, Gestalt, bass

\section{Introduction}

Karpinski (2000) describes "Gestalt hearing," identifying chords instantly and holistically, as the ideal endpoint of training in harmonic dictation. Rogers (2001) describes an incremental strategy to meet this goal, starting by asking students to identify cadences, then in later dictations gradually adding tonic and dominant expansions, followed by a series of predominants; this strategy is similar to the "chord-at-atime" approach taken in many current textbooks. Jarvis (2015) suggests a slightly different approach, using an analogy with language immersion to propose that students can develop "Gestalt" chord identification through extensive listening.

Yet it is not clear that Gestalt hearing - if it existswould operate in the ways Karpinski, Rogers, and Jarvis describe. After all, the subdominant chord (e.g.) is arguably not a single object but a collection of objects that differ in timbre, texture, inversion, spacing, function, and placement within a phrase. Principles from Gestalt psychology are also not clearly relevant here, as Karpinski describes Gestalt listening as emerging after an extended period of listening through other methods, while Gestalt psychologists asserted that perception of wholes is more immediate than perception of parts.

The study presented in this article was thus motivated by this question: does Gestalt hearing exist? That is, is Gestalt hearing actually a different mode of perception, learnable over time, that does not require other strategies such as listening for bass lines or chord qualities, or is it simply a way to describe using these other strategies efficiently and accurately in situationally-appropriate combinations? Subsidiary questions include: If this ability exists, what experiences and abilities are necessary in order to acquire it? Is this capacity available to everyone, or just to people with (e.g.) absolute pitch or extensive experience with a chord instrument?

Unfortunately, foundational experimental evidence about harmonic dictation is lacking. Butler (2000), reviewing the "Harmonic Dictation" portion of Karpinski (2000), lamented the lack of such data. But while scholars have suggested methods of instruction in the years since (Jarvis 2015; Sayrs 2019; Stevens 2016, 2020), there have been no studies about how individuals learn to hear harmony. Of course, these questions are difficult to address in a controlled, randomized study, both because of the difficulty of observing aural perception and because of the myriad of relevant intertwined variables that can be operational over large spans of an individual's life: level of absolute pitch, instruments played, years of experience, listening habits, working memory capacity, level of education, and more.

Facing the lack of existing experimental data on this subject, we conducted a survey in order to collect a wide variety of data that can be used to form hypotheses and support future controlled experiments. We approached the interpretation of this information as we might an experience with a student in the classroom: we made our best educated guesses, based on our knowledge and 
teaching experience. Nevertheless, more specific and controlled studies will be necessary in the future to confirm or disprove our preliminary observations and hypotheses.

\section{Method}

Our study, an anonymous online survey developed in Qualtrics, explored harmonic listening through a questionnaire about participants' abilities and musical experiences, three chord-identification exercises, and a self-reflection survey, resulting in both quantitative and qualitative data. We circulated the study through emails to aural skills classes at Utah State University and a post on the SMT Discuss online forum inviting other instructors to do the same at their own institutions.

\section{Participants}

73 participants completed the study; an additional 26 participants did not complete the entire study and are excluded from our results. The vast majority of participants reported being a current music major (58\%) or having completed a music major $(21 \%)$. The most common reported primary instrument was piano (34\%), followed by voice $(16 \%)$; the remaining participants were fairly evenly spread among a variety of wind, brass, and string instruments.

\section{Chord Identification Excerpts}

Participants were asked to identify the chords of several excerpts "in your own words," though example answers were given that suggested the desirability of Roman numerals. Three contrasting examples were chosen: a nearly-homophonic four-part excerpt composed explicitly for dictation and played on piano (Harmonic Dictation 42.6 from Karpinski 2017), a four-chord loop from a popular song (Beyoncé, "Halo"), and a short excerpt from a Classical piano sonata with an Alberti bass (the first two measures of Mozart, K. 332, mvmt. 2). The Karpinski and Mozart examples were embedded as .mp3s, and the Beyoncé as a Youtube video; each could be played and replayed at will.

\section{Participant Self-Reflections}

After the first chord identification exercise, and then again after the third, participants were asked to reflect on the process of identifying chords. First, they were asked to describe in their own words "how you typically identify a chord progression." Then they were asked to select the strategies they use from a provided list. These choices, distilled from a variety of textbooks that address harmonic dictation, included "Expectation of what is likely to come next," "Chord quality (major, minor, etc.)," "Bass line," "The feeling of a chord (the 'one-ness' or 'it feels dominant-y')," "Solfege syllables or scale degrees in the chords," "Cadences," "Imagining playing the chord on some instrument," and "Other."

\section{Scoring}

In order to categorize and compare participant results in the harmony identification section, we graded each participant's answer on each excerpt. To enable comparisons across examples, we set the maximum possible score for each at 100 percent; each chord in the excerpt, then, was worth $100 / C$ percentage points, where $C$ represents the number of chords in the excerpt. Incorrect inversions were given half credit, and a superfluous chord added to an otherwise correct progression also resulted in a deduction of half the points allotted to a correct chord for that excerpt. While most participants used Roman numerals to answer these questions, a few used other systems. Where these gave clear and specific answers (for example, one participant used notation such as " $1 / 3$ " to indicate a tonic chord in first inversion) we scored them similarly; where answers were vague or nonspecific, we left them out of our results. We also figured, for each participant, their average score across all three examples.

\section{Results}

Our survey participants had a wide range of average scores across the three chord-identification exercises (Figure 1). We analyzed the results through three lenses: first, demographic data; second, evidence from participant reflections on chord identification; and finally, corroboration from analysis of incorrect chord identifications.

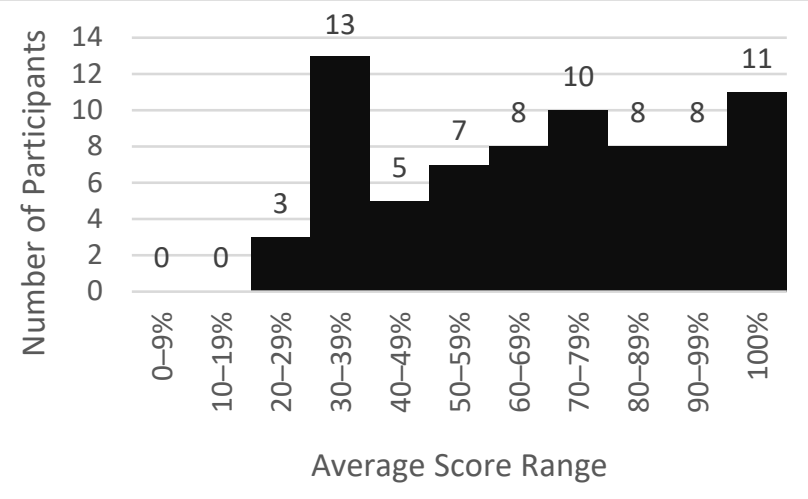

Figure 1: Histogram of participants' average scores (expressed as percent correct) across the three harmonic identification excerpts. 
Among demographic factors that may affect participants' chord-identifying abilities, we focused on three that seem to hold the potential for building conceptual models of chord Gestalts. First, presence of absolute pitch, including both Heightened Tonal Memory (HTM) and Absolute Pitch (AP; Ross et al. 2005; Figure 2). Second, playing a polyphonic primary instrument, though we tracked only piano because very few participants listed other polyphonic instruments as primary (Figure 3). Finally, years of formal lessons on the primary instrument (Figure 4) and years of formal lessons on piano regardless of primary instrument (Figure 5). Note that excluding the top-right-most two data points in Figure 4 would result in a less-sloped trend line, while in Figure 5 the outliers appear to be the two low-performing participants with 15 years of piano instruction.

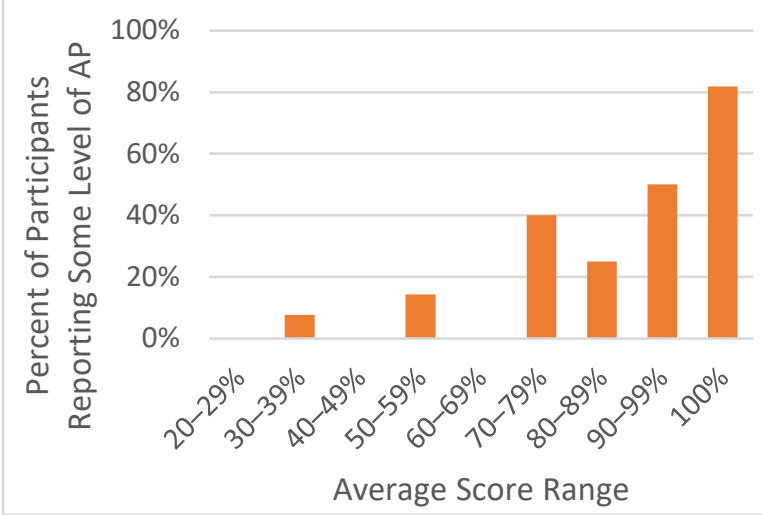

Figure 2: Percent of participants in each average-score category that reported some level of absolute pitch.

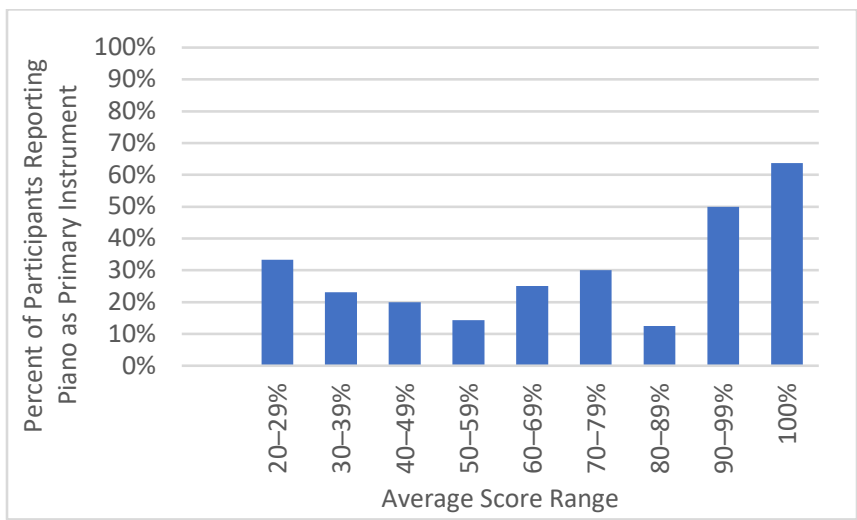

Figure 3: Percent of participants in each average-score category that reported piano as primary instrument.

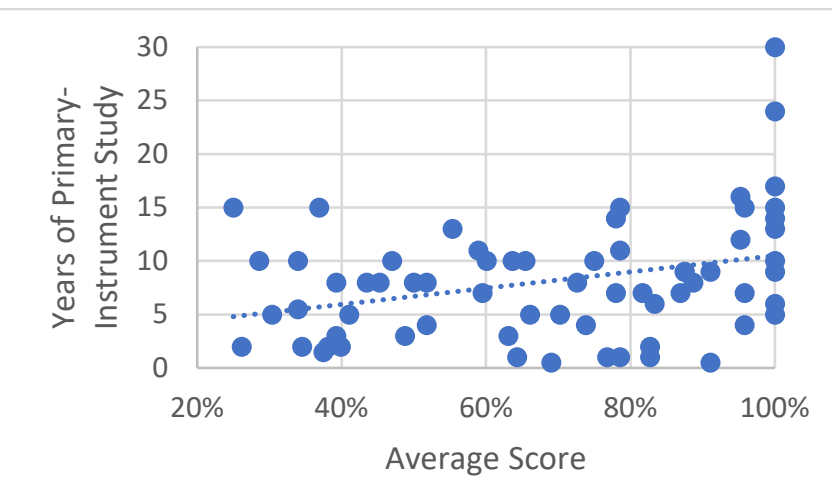

Figure 4: Scatterplot of average score on harmonic identification tasks against years of formal primaryinstrument study.

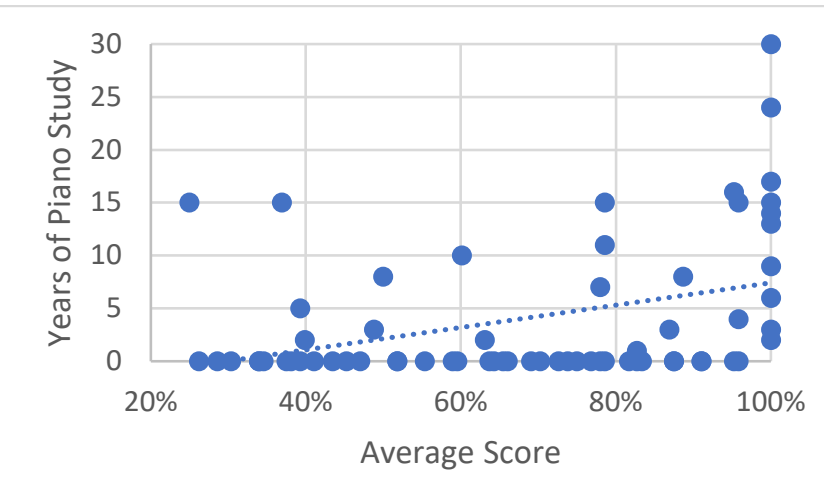

Figure 5: Scatterplot of average score on harmonic identification tasks against years of formal piano study.

When asked to select from a list those strategies that they use when identifying chords, $51 \%$ of participants selected "The feeling of the chord (the 'one-ness' or 'it feels dominant-y')," the choice intended to communicate Gestalt listening. Yet only "Imagining playing the chord on some instrument" was chosen by less participants, and several other strategies were far more popular (Figure 6). Even among the 11 participants who scored $100 \%$ across all three excerpts, who would presumably be those most likely to experience Gestalt hearing, only 55\% selected "The feeling of the chord" while $73 \%$ selected "Bass line." Participants' free-response reflections similarly tended to emphasize listening for the bass. 


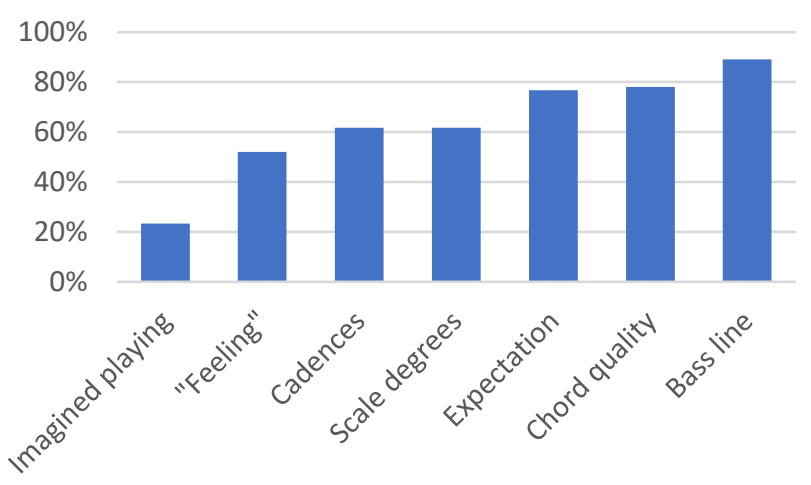

Figure 6: Percent of participants that indicated they use each chord-identification strategy.

Nevertheless, the free-response reflections of some participants who scored $100 \%$ did use wording that may suggest a truly different mode of (Gestalt) perception. Only two did not mention any other strategy: one, who has AP, wrote "I hear simultaneously in absolute pitches, Roman numeral function in relation to a local tonic, and figured bass symbols"; the other, who does not have AP, wrote, "I've internalized a kind of mental space for diatonic harmonies and outer-voice counterpoint, so I usually think about chord progressions traversing that space to identify them." Intriguingly, these were the two participants who reported the highest number of years of piano study (30 and 24, respectively). A few other participants among those who scored $100 \%$ combined similar statements with additional strategies; the one that most strongly suggested Gestalt hearing was "Sometimes, I can just tell instantly - other chords will require listening closely to individual notes within to figure them out." The remaining descriptions focused on component-based strategies such as "I follow the bass and try to identify its solfege, then I listen to the melody and its solfege."

Finally, common types of errors on the chordidentification exercises suggest that even highperforming chord identifiers rely heavily on an excerpt's bass line. For example, the most common error in the Karpinski dictation was labelling $\mathrm{ii}^{6}$ as IV, an error made even by half of the 8 participants whose average scores were between 90-99\%. The excerpt with the highest average score across all participants $(87.5 \%)$ was the Beyoncé song, in which all chords were in root position, while the lowest overall average score $(62.5 \%)$ was for the Mozart example, whose functional bass line was integrated into a potentially distracting Alberti bass. While incorrect answers in the examples with distinct bass lines (Karpinski and Beyoncé) typically preserved the correct bass contour, this was not true for the Mozart example. In fact, though our sample size is not large enough to establish this clearly, preliminary observations suggest that one of the primary factors that distinguished higher-performers (who scored an average of 80-99\%) from lower-performers (those with an average score of $<79 \%$ ) may have been the ability to successfully extract the bass line in the Mozart example.

\section{Discussion}

Drawing on these preliminary results, we propose a number of hypotheses and questions for future study.

First, with regard to our motivating question, we hypothesize that Gestalt hearing is not attainable as a primary mode of attending for most musicians at the college level. Nevertheless, there is tantalizing evidence that this kind of Gestalt hearing is available to some listeners, perhaps, as Karpinski (2000) suggests, as a result of other techniques practiced over "weeks, months, or even years" (p. 119). If so, then it is still an open question whether this is a skill available to all or whether it is only available to those with certain kinds of experiences or abilities. Notably, the two respondents whose wording strongly suggests Gestalt hearing reported 30 and 24 years (respectively) of formal piano instruction.

Second, we note that prevalence of AP/HTM, prevalence of piano as primary instrument, and years of formal piano instruction all appear to be higher among those who scored well at harmonic identification. We encourage further study to determine whether these are simply correlations or whether some combination significantly affects harmonic identification.

Third, we hypothesize that the most important strategy for chord identification is listening for bass lines. As noted above, this strategy was selected the most often by both our participant population as a whole and our highest-scoring group, and it was mentioned frequently in free responses. If this is true, then instruction in harmonic dictation would likely benefit from greater emphasis on bass-listening strategies. While current textbooks do often ask for bass- or outervoice dictation to accompany harmonic dictation, they also tend to organize their chapters/units around Roman numerals regardless of inversion/bass, and typically do not include advice on how to listen to bass lines. Ideas here might be derived from Sayrs 2019.

It is possible that our traditional method of judging harmonic hearing through Roman numerals with inversion symbols artificially inflated the apparent 
importance of listening for the bass; after all, to determine the correct inversion symbol, one must detect the bass. Nevertheless, participant free responses suggested that the bass was often used as a primary tool for detecting chords, not simply as a necessary step for determining inversions (e.g., "I listen for the bass note, and spell up the triad by note from that pitch to find the chord identity and inversion," and "I listened to the bass line, mostly, and thought about characteristic progressions in the common-practice style"). It is also likely that there is some circularity here: that the inversion of a chord (and thus its implied bass) is part of our traditional chord symbol because it is important to perception of chord identity. Still, it might be helpful for future research to examine whether musicians still listen for bass lines to this degree even when they are not asked to identify chord inversions.

One particularly ripe area for future study is the effects of formal instruction/experience with a chord instrument on harmonic listening. Again, our two participants who seemed to indicate Gestalt hearing had extensive piano experience, and piano experience was common among high-scoring participants. While this might suggest the importance of piano study to harmonic dictation skills, it is also possible that these participants benefitted from the presence of two excerpts in the study performed on piano, or that the prevalence of piano-based excerpts in current textbooks means that current teaching methods work best for pianists. In addition, it would be useful to study whether there are differences in how polyphonic-instrument players beyond pianists-guitarists, harpists, marimbists, etc.-perceive harmony; our sample size for each of these instruments was too small to suggest even preliminary conclusions.

\section{Conclusion}

This is the first relatively large-scale study of harmonic hearing, and as such, our focus has been on gathering a wide range of data that will be useful in planning future studies. Nevertheless, these data allow us to make some educated guesses about a few matters relevant to the teaching of this skill. In particular, our study suggests that "Gestalt hearing"-as appealing as it is - may be more complicated and less universally available than is sometimes assumed. If this is true, then the field of aural skills pedagogy might benefit from focusing instruction in harmonic listening more on strategies (particularly listening for bass lines, but also listening for chord qualities, characteristic inner-voice scale degrees, cadences, etc.) rather than on Roman numerals. We hope that future studies on harmonic listening follow, and we look forward to the results.

\section{References}

Butler, D. (2000). Review of Gary Karpinski, Aural Skills Acquisition: The Development of Listening, Reading, and Performing Skills in College-Level Musicians. Journal of Music Theory Pedagogy, 14, 105-114.

Jarvis, B. E. (2015). Hearing Harmony Holistically: Statistical Learning and Harmonic Dictation. Engaging Students: Essays in Music Pedagogy, 3. https://doi.org/10.18061/es.v3i0.7197

Karpinski, G. S. (2000). Aural Skills Acquisition. Oxford University Press.

Karpinski, G. S. (2017). Instructor's Dictation Manual for Manual for Ear Training and Sight Singing (2nd ed.). W.W. Norton.

Rogers, N. (2001). Review of Gary S. Karpinski, Aural Skills Acquisition: The Development of Listening, Reading, and Performing Skills in College-Level Musicians. Indiana Theory Review, 22(2), 83-93.

Sayrs, E. (2019). The Case For More Bass in the Aural Skills Curriculum. Pedagogy Into Practice: Teaching Music Theory in the Twenty-First Century, Santa Barbara.

Stevens, D. B. (2016). Symphonic Hearing: Mastering Harmonic Dictation Using the Do/Ti Test. Journal of Music Theory Pedagogy, 30, 111-176.

Stevens, D. B. (2020). In Search of Hidden Treasures. In The Routledge Companion to Music Theory Pedagogy (pp. 211-216). https://doi.org/10.4324/9780429505584-34
Routledge. 